\title{
Reduced Sensitivity to Non-Fear-Related Stimulus Changes in Panic Disorder
}

\author{
Johannes Rentzsch ${ }^{a, b}$ Lars Thoma ${ }^{a, c}$ Katharina Gaudlitz ${ }^{d}$ Nicole Tänzer ${ }^{a}$ \\ Jürgen Gallinat ${ }^{\mathrm{e}}$ Norbert Kathmann ${ }^{c}$ Andreas Ströhle ${ }^{\mathrm{a}}$ Jens Plag ${ }^{\mathrm{a}}$ \\ ${ }^{a}$ Department of Psychiatry and Psychotherapy, Campus Mitte, Charité - Universitätsmedizin Berlin, corporate \\ member of Freie Universität Berlin, Humboldt-Universität zu Berlin and Berlin Institute of Health, Berlin, Germany; \\ ${ }^{b}$ Department of Psychiatry, Psychotherapy and Psychosomatics, Brandenburg Medical School Theodor Fontane, \\ Neuruppin, Germany; ' Department of Psychology, Humboldt-Universität zu Berlin, Berlin, Germany;

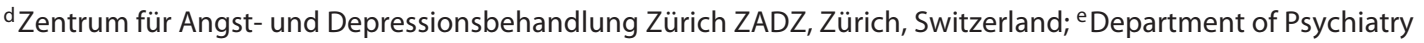 \\ and Psychotherapy, University Medical Center Hamburg-Eppendorf (UKE), Hamburg, Germany
}

\section{Keywords}

Electroencephalography · Mismatch negativity ·

Preattentive information processing · Anxiety disorders

\begin{abstract}
Panic disorder (PD) is associated with increased body vigilance and reduced cognitive resources directed at non-fear-related stimuli, particularly in the absence of stimulus-rich environments. To date, only few studies have investigated whether this deficit in PD is reflected in reduced mismatch negativity (MMN), an event-related potential indexing preattentive sensitivity to unexpected stimulus changes. We tested 35 patients affected by PD and 42 matched healthy controls in an oddball paradigm, using frequency and duration deviant stimuli to measure auditory MMN. PD patients displayed reduced duration MMN amplitudes in comparison to healthy controls. No group differences were detected for duration MMN latency, as well as frequency MMN indices. Results support the notion of reduced processing of non-fear-related stimuli in PD patients, particu-
\end{abstract}

\section{KARGER}

(c) 2019 S. Karger AG, Basel

E-Mail karger@karger.com

www.karger.com/nps larly with regard to the preattentive processing of sound duration deviants. Additionally, our findings are in line with clinical studies reporting divergent deficits in preattentive processing of frequency and duration deviants.

(c) 2019 S. Karger AG, Basel

\section{Introduction}

Panic disorder (PD) is a condition characterized by recurrent panic attacks, worry about their further occurrences and avoidance behaviors [1]. Its lifetime prevalence is estimated to range between 3 and 4\% [2]. It is associated with both significant impairment in quality of life $[3,4]$ and substantial financial cost [5].

On a cognitive level, PD is associated with abnormalities in attention control and information processing. In gen-

J.R. and L.T. contributed equally to this paper. 
eral, evidence shows that PD patients preferentially direct their attention to threat-related stimuli such as body perceptions or panic-related stimuli (such as crowds). In contrast to these threat-related stimuli, PD patients have been shown to process their environment to a reduced extent [6-9]. This attentional bias is assumed to lead to a pronounced perception of early panic symptoms which in turn leads to increased alertness and thereby further intensifies panic attacks [10]. Additionally, this attentional bias seems to maintain $\mathrm{PD}$ as increased body vigilance predicts the future occurrence of panic attacks [11] and because it has been shown that increased awareness of body symptoms in $\mathrm{PD}$ is associated with a higher reactivity to sudden fear-inducing environmental stimuli [12].

It needs to be noted however that abnormalities in information processing in PD cannot be simplified as increased processing of body and anxiety symptoms while showing reduced processing of environmental non-fear-related stimuli. In particular, studies have reported deficits in PD to ignore monotonous serial stimuli [13] or stimuli of low relevance $[12,14-16]$. Hence, it is more accurate to assume that PD patients show deficits in adequately assessing the meaning of stimuli, that is differentiating signal from noise [17], and therefore misdirect information-processing resources.

Neuropsychological studies of event-related potentials (ERP) support this notion of abnormal information processing in $\mathrm{PD}$, although it needs to be noted that findings are at times mixed. For instance, one branch of ERP studies investigated N1. This amplitude is observed in early attentive information processes and declines when stimuli are repeatedly displayed $[18,19]$. There is evidence for increased N1 in PD [20-23], though some studies did report equal N1 amplitudes between PD patients and healthy controls (HC) [24, 25]. Further abnormalities in information processing in $\mathrm{PD}$ have been reported for increased $\mathrm{P} 3 \mathrm{a}$ amplitudes to rare stimuli in a common two-tone oddball task, most likely indicating irregularities in passive attention processes [24]. In addition, reduced P3 amplitudes have been reported, equally hinting at deviations in active attention processes [26]. Additionally, there is evidence for increased error-related negativity in PD patients which suggests a tendency for increased processing of internal signals after committing errors [27]. Taken together, there is substantial neuropsychological evidence for information-processing abnormalities in PD.

One possibility to further elucidate abnormalities in the processing of non-fear-related stimuli in PD is the mismatch negativity (MMN). MMN is an ERP that commonly peaks between 150 and $250 \mathrm{~ms}$ after an unexpected stimulus change. The MMN is defined as the difference wave calcu- lated by subtracting the ERP to standard stimuli from the ERP to deviant stimuli. Its latency becomes shorter when stimuli changes are more salient $[28,29]$. As the MMN can be detected even when the stimuli are not consciously attended to, it is interpreted as a preattentive cognitive process indexing the accuracy in detecting changes [30]. Traditionally, the MMN has been studied with electroencephalography (EEG) for simple auditory stimuli. A very common experimental paradigm is the passive oddball paradigm. It consists in the presentation of standard stimuli and less frequent stimuli that differ in certain characteristics such as length or frequency from the standard stimuli. However, a considerable amount of research has replicated MMN across different modalities [e.g., visual stimuli 31], increasingly complex stimulus configurations [for instance grammar violations, 32] and various methodologies [such as functional magnetic resonance imaging, 33]. These varying replications suggest that $\mathrm{MMN}$ represents a universally important preattentive cognitive process that applies to detection of changes across various modalities.

MMN has been used with increasing interest in order to elucidate the neurocognitive underpinnings of distinct clinical conditions [34]. Most prominently, evidence for the association of cognitive deficits with reduced MMN amplitudes has been found in dyslexia [35], schizophrenia [36-40], depression [41] and Alzheimer's disease [42]. To date, relatively few studies have investigated MMN in PD. To our knowledge, only two trials available in English addressed this topic in small samples of up to 15 patients with PD $[43,44]$, yielding contrasting results. Tang et al. [44] found decreased MMN amplitudes by using a visual paradigm, one other study applying auditory stimuli reported increased intensity MMN but normal frequency and duration MMN amplitudes in this condition [43].

Hence, the present study sought to clarify the characteristics of MMN in PD within a relatively large sample of patients. We hypothesized abnormalities in MMN in PD as compared to HC. As evidence to date on MMN in PD is inconsistent, the direction of this effect remains difficult to estimate.

\section{Method}

Participants and Procedure

We tested 35 patients with PD and $42 \mathrm{HC}$ subjects. This sample was part of a more extended trial focusing on the effect of aerobic exercise on cognitive behavioral therapy in $\mathrm{PD}$ that was published elsewhere [45]. It was approved by the ethics committee at Charité - Universitätsmedizin Berlin (EA1/129/08) and registered at
Rentzsch/Thoma/Gaudlitz/Tänzer/ Gallinat/Kathmann/Ströhle/Plag 
Table 1. Demographic and clinical characteristics of the sample (with comparison statistics, $t$ test and $\chi^{2}$ test)

\begin{tabular}{llll}
\hline & $\begin{array}{l}\text { PD group } \\
(n=35)\end{array}$ & $\begin{array}{l}\text { HC group } \\
(n=42)\end{array}$ & Comparison statistic \\
\hline Sex, female & $15.0(43 \%)$ & $16.0(38 \%)$ & $\chi^{2}(1)=0.18 ; p>0.6$ \\
Right-handedness & $32.0(91 \%)$ & $40.0(95 \%)$ & $\chi^{2}(1)=0.59 ; p>0.7$ \\
Age, years & $31.5(6.9)$ & $34.1(9.8)$ & $t(75)=1.30 ; p>0.19$ \\
Age of onset, years & $28.7(7.8)$ & & \\
Duration of illness, months & $42.1(55.5)$ & $15.9(2.8)$ & $t(75)=1.217 ; p>0.2$ \\
Time of EEG & $15.1(3.2)$ & $2.1(2.8)$ & $t(39.06)=10.41 ; p<0.0001$ \\
HAM-A & $19.1(9.3)$ & $1.7(2.1)$ & $t(36.34)=9.41 ; p<0.0001$ \\
BAI & $18.1(10.2)$ & $0.1(0.3)$ & $t(34.1)=12.31 ; p<0.0001$ \\
PAS & $18.7(8.9)$ & $0.5(0.9)$ & $t(36.07)=8.31 ; p<0.0001$ \\
HAMD-17 & $6.8(4.5)$ & $1.6(2.1)$ & $t(37.26)=6.84 ; p<0.0001$ \\
BDI-II & $12.0(9.0)$ & &
\end{tabular}

Values are presented as $n$ (\%) or means (standard deviations). HAM-A, Hamilton Anxiety Rating Scale; BAI, Beck Anxiety Inventory; PAS, Panic and Agoraphobia Scale; HAMD-17, Hamilton Rating Scale for Depression; BDI-II, Beck Depression Inventory II.

"ClinicalTrials.gov" (identifier: NCT01788800). All study procedures were conducted in accordance with the Declaration of Helsinki.

Patients were recruited through the Charités outpatient clinic for anxiety disorders. PD was diagnosed by a trained psychologist via the Mini-International Neuropsychiatric Interview [46]. HC subjects were recruited through advertisement in the clinic's environment. All participants provided written informed consent prior to their participation. We included patients and $\mathrm{HC}$ aged between 18 and 70 years, with no history of head trauma or neurological disease. Further inclusion criteria for patients encompassed a diagnosis of PD according to the Diagnostic and Statistical Manual of Mental Disease, 4th revision, DSM-IV [47], absence of pharmacotherapy within the last 4 weeks as well as the absence of any other severe psychiatric diagnosis or acute suicidality. HC were included if they were free of any present psychiatric diagnosis and did not consume drugs or psychotropic medication. Anxiety symptom severity in both patients and HC was assessed using the Hamilton Anxiety Scale [HAMA, 48], the self-rating version of the Panic and Agoraphobia Scale [PAS, 49] and the Beck Anxiety Inventory [BAI, 50]. The Hamilton Depression Scale [HAMD-17, 51] and the Beck Depression Inventory II [BDI-II, 52] were used to measure depressive symptoms.

Demographic and clinical characteristics of our sample are presented in Table 1. PD patients and HC did not differ significantly with regard to age, sex ratio and handedness. As expected, patients reported significantly more anxiety- and depression-related symptoms than control subjects.

\section{Auditory Stimulation}

The participants were seated in a chair with a head rest in a sound-attenuated room. Auditory stimuli were presented binaurally through headphones. The participants were instructed to keep their eyes open and look at a fixation point on a screen. In total, 1,800 tone stimuli ( $80 \mathrm{~dB}$ SPL) were presented. Standard tones $(80 \%)$ had a frequency of $1,000 \mathrm{~Hz}$ and duration of $90 \mathrm{~ms}$
(10 $\mathrm{ms}$ rise/fall time). In contrast, frequency deviant tones (10\%) had a frequency of $1,200 \mathrm{~Hz}$ and duration deviant tones a duration of $50 \mathrm{~ms}$ (10 ms rise/fall time). Deviant tones were always followed by a standard tone. Intertrial intervals varied in a pseudorandomized sequence between 350 and 650 ms (mean: $500 \pm$ $110 \mathrm{~ms}$ ).

\section{Electroencephalographic Data}

EEG was carried out with $32 \mathrm{Ag} / \mathrm{Ag}-\mathrm{Cl}$ electrodes according to the extended international 10/20 system using an electrode cap (EASYCAP GmbH, Herrsching, Germany). The ocular electrode was located on the outer canthus of the left eye and the ground electrode on the forehead. Electrode impedances were below $10 \mathrm{k} \Omega$. EEG was recorded with BrainAmp hardware (Brain Products $\mathrm{GmbH}$, Munich, Germany) with a sampling rate of $250 \mathrm{~Hz}$ (high-pass filtered at $0.016 \mathrm{~Hz}$ ) and referenced to electrode $\mathrm{Cz}$. Offline MMN analysis was conducted with Brain Vision Analyzer 2.0 (Brain Products, Munich, Germany) and EEGlab Version v13.1.1 [53]. Artifacts were automatically corrected using the eeglab-plugins Artifact Subspace Reconstruction Version 0.13 [54] and Automatic Artifact Removal Version 1.3 [55] for short-term artifacts, e.g. ocular movements and electrocardiac artifacts. After filtering (high pass: $0.5 \mathrm{~Hz}$, order 2; 50 $\mathrm{Hz}$ notch filter; zero phase shift Butterworth IIR filter) and artifact rejection $(100 \mu \mathrm{V})$, data were re-referenced to common average and $30-\mathrm{Hz}$ low-pass filtered (order 2). Data were segmented (-150 to 300 $\mathrm{ms}$ ), baseline corrected ( -150 to $0 \mathrm{~ms}$ ) and averaged per subject and stimulus condition. MMN were obtained by subtracting the waveforms elicited by standards from those elicited by deviants at $\mathrm{Cz}$ and Fz electrodes. MMN amplitudes were defined as the most negative peak from 170 to $270 \mathrm{~ms}$ (duration MMN) and 110 to $240 \mathrm{~ms}$ (frequency MMN) according to the grand average. MMN were extracted as negative mean amplitude (10 $\mathrm{ms}$ around the peak).

\section{Statistical Analysis}

MMN amplitudes and latencies were analyzed with a repeated measurement multivariate analysis of covariance (MANCOVA) 
with the between-subject factors sex and group (PD and control), as well as the within-subject factor electrode ( $\mathrm{Fz}$ and $\mathrm{Cz})$. Additionally, age was analyzed as covariate.

Correlations between the duration MMN and anxiety severity, as well as general somatic symptoms "HAMA-somatic anxiety subscore" (calculated as the sum of HAMA items 7-10 measuring general muscular somatic symptoms, general sensory somatic symptoms, cardiovascular symptoms and respiratory symptoms) were analyzed with Pearson correlations. An $\alpha$-level of 0.05 indicated significance in all analyses.

\section{Results}

MMN latencies and amplitudes at $\mathrm{Cz}$ and $\mathrm{Fz}$ electrodes are given in Table 2. Additionally, evoked potentials for the standard stimuli, the duration and frequency deviants are displayed in Figure 1.

Repeated measurement MANCOVA for MMN latency revealed no significant effect of the between-subject factor group $(F(2,72)=0.39, p>0.531)$ and of the between-subject factor sex $(F(2,72)=0.262, p>0.770)$. The withinsubject factor electrode $(\mathrm{Fz}, \mathrm{Cz} ; F(2,72)=0.242, p>0.786)$ had no significant effect. There was a statistical trend for an effect of the covariate age $(F(2,72)=2.850, p<0.07)$.

Repeated measurement MANCOVA for MMN amplitudes revealed a significant effect of the between-subject factor group $(F(2,72)=3.863, p<0.026)$, but not of the between-subject factor $\operatorname{sex}(F(2,72)=3.10, p>0.73)$ and the within-subject factor electrode $(\mathrm{Fz}, \mathrm{Cz} ; F(2,72)=$ $1.782, p>0.17)$ on MMN. The covariate age had no statistically significant impact on $\operatorname{MMN}(F(2,72)=1.134$, $p>0.32$ ). Post hoc ANCOVA showed a significant effect of the main factor diagnosis on duration MMN amplitude $(F(1,73)=7.32, p<0.009)$ but not on frequency MMN amplitude $(F(1,73)=0.76, p>0.78)$.

The duration MMN amplitude difference remains significant when patients with strong depressive symptoms $(n=3$, as defined by a HAMD-17 score $>12)$ are excluded from analysis, $(F(1,70)=6.401, p<0.014)$. The significant differences for duration $\mathrm{MMN}$ amplitude remain also significant under exclusion of all left-handed participants in the patient group $(n=3)$ and the HC group $(n=2)$ $(F(1,68)=7.166, p<0.01)$.

There were no statistically significant correlations between the duration MMN amplitude and severity of anxiety symptoms (HAMA, $r=0.01, p>0.9$; PAS, $r=-0.17$, $p>0.3$; BAI, $r=-0.04, p>0.8$ ) or depressive symptoms (HAMD-17, $r=-0.08, p>0.6$; BDI-II, $r=0.05, p>0.7$ ). However, the correlation between "HAMA-somatic anxiety subscore" items 7-10 measuring somatic symptoms reached significance: $r=0.36, p=0.04$.
Table 2. MMN latency (ms) and amplitude $(\mu \mathrm{V})$ for panic disorder patients and healthy control group at $\mathrm{Cz}$ and $\mathrm{Fz}$ electrodes

\begin{tabular}{|c|c|c|c|c|}
\hline & \multicolumn{2}{|c|}{$\begin{array}{l}\text { PD group } \\
(n=35)\end{array}$} & \multicolumn{2}{|c|}{$\begin{array}{l}\text { HC group } \\
(n=42)\end{array}$} \\
\hline & mean & $\mathrm{SD}$ & mean & $\mathrm{SD}$ \\
\hline \multicolumn{5}{|l|}{ Latency, ms } \\
\hline $\mathrm{Cz}$ frequency & 175 & 37 & 167 & 38 \\
\hline Fz frequency & 178 & 31 & 179 & 37 \\
\hline $\mathrm{Cz}$ duration & 221 & 23 & 225 & 22 \\
\hline Fz duration & 222 & 26 & 224 & 25 \\
\hline \multicolumn{5}{|l|}{ Amplitude, $\mu V$} \\
\hline $\mathrm{Cz}$ frequency & -0.95 & 0.59 & -0.97 & 0.71 \\
\hline Fz frequency & -1.37 & 0.86 & -1.4 & 0.93 \\
\hline $\mathrm{Cz}$ duration & -0.64 & 0.44 & -0.92 & 0.63 \\
\hline Fz duration & -0.82 & 0.56 & -1.17 & 0.79 \\
\hline
\end{tabular}

Given are means and standard deviations.

\section{Discussion}

This study investigated MMN as a preattentive indicator for sensitivity to non-fear-related stimulus changes in unmedicated patients with PD. Most importantly, we found significant lower duration MMN amplitudes in the PD group compared to the HC group. No significant group difference was detected for frequency MMN amplitudes, as well as frequency MMN latencies and duration MMN latencies.

This finding agrees to some extent with a study conducted by Tang et al. [44]. They investigated visual $\mathrm{MMN}$ related to negative and positive facial expressions in $12 \mathrm{PD}$ patients and $17 \mathrm{HC}$ and found significantly attenuated visual MMN in response to both conditions in PD patients as compared to HC. The authors interpret this difference as a deficit in PD patients to automatically detect facial emotions. In a synopsis, these results suggest an information-processing impairment in PD which can be found for both more complex stimuli (such as faces) and less complex stimuli (such as sounds). However, one other finding from the same study group indicates no difference in duration and frequency MMN amplitudes between PD patients and HC, but significantly higher sound intensity and location MMN amplitudes in PD patients as compared to HC [43]. Apart from the small sample size of $15 \mathrm{PD}$ patients in their study, one potential explanation of these divergent results might be the use of a different paradigm including five different deviants in the study by Chang et al. [43]. 
Possibly, this paradigm which includes a broader variety of stimuli simulates a rather stimulus-rich and therefore potentially more fear-related environment than the use of a more reduced paradigm such as applied in the present study.

Considering these aspects, our results may support the notion of reduced preattentive sensitivity to nonfear-related stimulus changes in patients with PD. These results could potentially be explained by disturbed signal-to-noise discrimination in PD [17]. More precisely, PD patients display increased processing of stimuli that are either fear-related or erroneously categorized as fear-relevant and decreased information processing of remaining stimuli. Hence, reduced duration MMN amplitudes found in this study seem to underpin neurocognitive processes that are attenuated in PD patients, most likely due to the limited nature of cognitive resources. This interpretation is in line with prominent theoretical models of anxiety stating that anxiety leads to a reduced attention focus to fear-unrelated stimuli [56].

The finding of reduced duration but not frequency MMN amplitudes in PD patients might be explained by different aspects. One explanation for this finding might be that processing of sound duration deviants is affected to a larger degree than the processing of sound frequency deviants in PD patients. This explanation is supported by studies showing divergent deficits in duration and frequency MMN amplitudes in other psychiatric conditions $[57,58]$, likely due to differences in the underlying neuronal networks involved in preattentive deviant sound processing. While frequency deviants seem to be processed in auditory-temporal brain areas, duration deviant processing seems to involve further key brain areas forming greater and more distributed networks [59]. Hence, normal frequency but disturbed duration MMN amplitudes in $\mathrm{PD}$, as seen in our study, may indicate more complex disturbed network activity than simple auditory-temporal brain dysfunction in preattentive automatic information processing. One further explanation might be that duration deviants likely have a larger reliability than frequency deviants [60] and therefore are more sensitive to group differences.

We found no correlations between duration MMN and severity of PD in our sample. This is in line with the trial of Tang et al. [44] who did not detect an association between MMN amplitude and symptom severity either. In contrast, Chang et al. [43] found an association between MMN amplitude and symptom severity as in- dexed by the panic disorder severity scale [61]. This divergent finding is potentially due to the use of a more complex design in Chang and colleagues' study, simulating a rather stimulus-rich environment that is potentially more closely related to an increased level of stimulus perception in panic attacks. Yet, in the present study, the correlation between a HAMA subscore calculated for somatic symptoms and duration MMN amplitude reached significance. While this finding needs to be interpreted cautiously, it hints at the possibility that an increased perception of somatic symptoms is associated with fewer cognitive resources directed at preattentive processing of non-fear-related stimuli. As increased body vigilance has been repeatedly found in PD patients [8], this interpretation is in line with prominent theoretical models of anxiety stating that anxiety leads to a reduced attention focus to fear-unrelated stimuli [56].

As a limitation of our study, it needs to be noted that no patients with comorbidities and medication were included in this study. While this exclusion increases internal validity, however, it may reduce clinical representativeness of the sample. Additionally, there was no measure included for body vigilance. A strength of the present study is the relatively large sample size. Compared to former studies addressing this topic $[43,44]$, we included more than twice as many patients and HC. This enhances both the validity and the generalizability of our results.

\section{Acknowledgment}

We thank Charité - Universitätsmedizin Berlin for providing the required resources for the present study.

\section{Statement of Ethics}

Patients participating in this study have given their written informed consent. This study protocol has been approved by the ethicscommitteeatCharité-Universitätsmedizin Berlin(EA1/129/08) and registered at "ClinicalTrials.gov" (identifier: NCT01788800). All study procedures were conducted in accordance with the Declaration of Helsinki.

\section{Disclosure Statement}

The authors have no conflict of interest to declare. 


\section{References}

1 American Psychiatric Association. Diagnostic and statistical manual of mental disorders. 5th ed. Washington: American Psychiatric Association; 2013.

2 Wittchen HU, Jacobi F, Rehm J, Gustavsson A, Svensson M, Jönsson B, et al. The size and burden of mental disorders and other disorders of the brain in Europe 2010. Eur Neuropsychopharmacol. 2011 Sep;21(9):655-79.

3 Lochner C, Mogotsi M, du Toit PL, Kaminer D, Niehaus DJ, Stein DJ. Quality of life in anxiety disorders: a comparison of obsessivecompulsive disorder, social anxiety disorder, and panic disorder. Psychopathology. 2003 Sep-Oct;36(5):255-62.

4 Olatunji BO, Cisler JM, Tolin DF. Quality of life in the anxiety disorders: a meta-analytic review. Clin Psychol Rev. 2007 Jun;27(5): 572-81.

5 Olesen J, Gustavsson A, Svensson M, Wittchen HU, Jönsson B; CDBE2010 study group; European Brain Council. The economic cost of brain disorders in Europe. Eur J Neurol. 2012 Jan;19(1):155-62.

6 Hayward P, Ahmad T, Wardle J. Attention to bodily sensations: a test of the cognitive-attentional model of panic. Depress Anxiety. 2000;12(4):203-8.

7 Kállai J, Kóczán G, Szabó I, Molnár P, Varga J. An experimental study to operationally define and measure spatial orientation in panic agoraphobic subjects, generalized anxiety and healthy control groups. Behav Cogn Psychother. 1995;23(02):145-52.

8 Schmidt NB, Lerew DR, Trakowski JH. Body vigilance in panic disorder: evaluating attention to bodily perturbations. J Consult Clin Psychol. 1997 Apr;65(2):214-20.

9 Wells A, Papageorgiou C. The observer perspective: biased imagery in social phobia, agoraphobia, and blood/injury phobia. Behav Res Ther. 1999 Jul;37(7):653-8.

10 Clark DM, Salkovskis PM, Öst LG, Breitholtz E, Koehler KA, Westling BE, et al. Misinterpretation of body sensations in panic disorder. J Consult Clin Psychol. 1997 Apr;65(2): 203-13.

11 McNally RJ. Anxiety sensitivity and panic disorder. Biol Psychiatry. 2002 Nov;52(10):93846.

12 Ludewig S, Geyer MA, Ramseier M, Vollenweider FX, Rechsteiner E, Cattapan-Ludewig K. Information-processing deficits and cognitive dysfunction in panic disorder. J Psychiatry Neurosci. 2005 Jan;30(1):37-43.

13 Ghisolfi ES, Heldt E, Zanardo AP, Strimitzer IM Jr, Prokopiuk AS, Becker J, et al. P50 sensory gating in panic disorder. J Psychiatr Res. 2006 Sep;40(6):535-40.

14 Kampman M, Keijsers GP, Verbraak MJ, Näring G, Hoogduin CA. The emotional Stroop: a comparison of panic disorder patients, obsessive-compulsive patients, and normal controls, in two experiments. J Anxiety Disord. 2002;16(4):425-41.
15 van den Heuvel OA, Veltman DJ, Groenewegen HJ, Witter MP, Merkelbach J, Cath DC, et al. Disorder-specific neuroanatomical correlates of attentional bias in obsessive-compulsive disorder, panic disorder, and hypochondriasis. Arch Gen Psychiatry. 2005 Aug. 62(8):922-33.

16 Windmann S, Sakhavat Z, Kutas M. Electrophysiological evidence reveals affective evaluation deficits early in stimulus processing in patients with panic disorder. J Abnorm Psychol. 2002 May;111(2):35769.

17 Gordon E, Liddell BJ, Brown KJ, Bryant R, Clark CR, Das P, et al. Integrating objective gene-brain-behavior markers of psychiatric disorders. J Integr Neurosci. 2007 Mar;6(1): $1-34$.

18 Budd TW, Barry RJ, Gordon E, Rennie C, Michie PT. Decrement of the N1 auditory event-related potential with stimulus repetition: habituation vs. refractoriness. Int J Psychophysiol. 1998 Dec;31(1):51-68.

19 Sambeth A, Maes JH, Quian Quiroga R, Coenen AM. Effects of stimulus repetitions on the event-related potential of humans and rats. Int J Psychophysiol. 2004 Aug;53(3): 197-205.

20 Iwanami A, Isono H, Okajima Y, Kamijima K. Auditory event-related potentials in panic disorder. Eur Arch Psychiatry Clin Neurosci. 1997;247(2):107-11

21 Knott V, Lapierre YD, Fraser G, Johnson N. Auditory evoked potentials in panic disorder. J Psychiatry Neurosci. 1991 Nov;16(4):21520.

22 Ogura C. Cognitive evoked potentials in psychiatric disorders. Electroencephalography and Clinical Neurophysiology. Electromyogr Motor Control. 1995;97(4):S48.

23 Wise V, McFarlane AC, Clark CR, Battersby M. Event-related potential and autonomic signs of maladaptive information processing during an auditory oddball task in panic disorder. Int J Psychophysiol. 2009 Oct;74(1): 34-44.

24 Clark CR, McFarlane AC, Weber DL, Battersby M. Enlarged frontal P300 to stimulus change in panic disorder. Biol Psychiatry. 1996 May;39(10):845-56.

25 Wang J, Miyazato H, Randall M, Hokama H, Hiramatsu K, Ogura C. The N200 abnormalities of auditory event-related potentials in patients with panic disorder. Prog Neuropsychopharmacol Biol Psychiatry. 2003 Sep; 27(6):1013-21.

26 Gordeev SA. Clinical-psychophysiological studies of patients with panic attacks with and without agoraphobic disorders. Neurosci Behav Physiol. 2008 Jul;38(6):633-7.

27 Valt C, Huber D, Erhardt I, Stürmer B. Internal and external signal processing in patients with panic disorder: an event-related potential (ERP) study. PLoS One. 2018 Nov; 13(11):e0208257.
28 Amenedo E, Escera C. The accuracy of sound duration representation in the human brain determines the accuracy of behavioural perception. Eur J Neurosci. 2000 Jul;12(7):2570-4.

29 Sams M, Paavilainen P, Alho K, Näätänen R. Auditory frequency discrimination and event-related potentials. Electroencephalogr Clin Neurophysiol. 1985;62:437-448.

30 Näätänen R, Paavilainen P, Rinne T, Alho K The mismatch negativity (MMN) in basic research of central auditory processing: a review. Clin Neurophysiol. 2007 Dec;118(12): 2544-90.

31 Alho K, Woods DL, Algazi A, Näätänen R. Intermodal selective attention. II. Effects of attentional load on processing of auditory and visual stimuli in central space. Electroencephalogr Clin Neurophysiol. 1992 May;82(5): 356-68.

32 Pulvermüller F, Shtyrov Y. Automatic processing of grammar in the human brain as revealed by the mismatch negativity. Neuroimage. 2003 Sep;20(1):159-72

33 Molholm S, Martinez A, Ritter W, Javitt DC, Foxe JJ. The neural circuitry of pre-attentive auditory change-detection: an fMRI study of pitch and duration mismatch negativity generators. Cereb Cortex. 2005 May;15(5):54551.

34 Schall U. Is it time to move mismatch negativity into the clinic? Biol Psychol. 2016 Apr;116: 41-6.

35 Schulte-Körne G, Deimel W, Bartling J, Remschmidt H. Auditory processing and dyslexia: evidence for a specific speech processing deficit. Neuroreport. 1998 Jan;9(2): 337-40.

36 Umbricht D, Krljes S. Mismatch negativity in schizophrenia: a meta-analysis. Schizophr Res. 2005 Jul;76(1):1-23.

37 Hayakawa YK, Kirino E, Shimoji K, Kamagata $\mathrm{K}$, Hori $\mathrm{M}$, Ito $\mathrm{K}$, et al. Anterior cingulate abnormality as a neural correlate of mismatch negativity in schizophrenia. Neuropsychobiology. 2013;68(4):197-204.

38 Horikoshi S, Shiga T, Hoshino H, Ochiai H, Kanno-Nozaki K, Kanno K, et al. The relationship between mismatch negativity and the COMTVal108/158Met genotype in schizophrenia. Neuropsychobiology. Epub 2018 Oct 16.

39 Inami R, Kirino E, Inoue R, Suzuki T, Arai H Nicotine effects on mismatch negativity in nonsmoking schizophrenic patients. Neuropsychobiology. 2007;56(2-3):64-72.

40 Kirino E, Inoue R. Relationship of mismatch negativity to background EEG and morphological findings in schizophrenia. Neuropsychobiology. 1999;40(1):14-20.

41 Umbricht D, Koller R, Schmid L, Skrabo A, Grübel C, Huber T, et al. How specific are deficits in mismatch negativity generation to schizophrenia? Biol Psychiatry. 2003 Jun; 53(12):1120-31. 
42 Pekkonen E. Mismatch negativity in aging and in Alzheimer's and Parkinson's diseases. Audiol Neurotol. 2000 May-Aug;5(3-4):216-24.

43 Chang Y, Xu J, Pang X, Sun Y, Zheng Y, Liu $Y$. Mismatch negativity indices of enhanced preattentive automatic processing in panic disorder as measured by a multi-feature paradigm. Biol Psychol. 2015 Feb;105:77-82.

44 Tang D, Xu J, Chang Y, Zheng Y, Shi N, Pang $\mathrm{X}$, et al. Visual mismatch negativity in the detection of facial emotions in patients with panic disorder. Neuroreport. 2013 Mar;24(5):20711.

45 Gaudlitz K, Plag J, Dimeo F, Ströhle A. Aerobic exercise training facilitates the effectiveness of cognitive behavioral therapy in panic disorder. Depress Anxiety. 2015 Mar;32(3): 221-8.

46 Hergueta T, Baker R, Dunbar GC. The MiniInternational Neuropsychiatric Interview (MINI): the development and validation of a structured diagnostic psychiatric interview for DSM-IV and ICD-10. J Clin Psychiatry. 1998;59:2233.

47 American Psychiatric Association. Diagnostic and statistic manual of mental disorders. Washington (DC): American psychiatric association; 1994.

48 Hamilton $\mathrm{M}$. The assessment of anxiety states by rating. Br J Med Psychol. 1959;32(1):50-5.
49 Bandelow B. Assessing the efficacy of treatments for panic disorder and agoraphobia. II. The Panic and Agoraphobia Scale. Int Clin Psychopharmacol. 1995 Jun;10(2):73-81.

50 Beck AT, Epstein N, Brown G, Steer RA. An inventory for measuring clinical anxiety: psychometric properties. J Consult Clin Psychol. 1988;56(6):893-97.

51 Hamilton M. A rating scale for depression. J Neurol Neurosurg Psychiatry. 1960 Feb; 23(1):56-62.

52 Beck AT, Steer RA, Brown GK. Beck depression inventory-II. San Antonio: Psychological Corporation; 1996. Vol 78, p. 490-98.

53 Delorme A, Makeig S. EEGLAB: an open source toolbox for analysis of single-trial EEG dynamics including independent component analysis. J Neurosci Methods. 2004 Mar; 134(1):9-21.

54 Mullen T, Kothe C, Chi YM, Ojeda A, Kerth $\mathrm{T}$, Makeig S, et al. Real-time modeling and 3D visualization of source dynamics and connectivity using wearable EEG. Engineering in Medicine and Biology Society (EMBC) 2013 , 35th Annual International Conference of the IEEE, Osaka, 2013, p. 2184-87.

55 Gómez-Herrero G, De Clercq W, Anwar H, Kara O, Egiazarian K, Van Huffel S, et al. Automatic removal of ocular artifacts in the EEG without an EOG reference channel. NORSIG 2006, Proceedings of the 7th Nordic Signal Processing Symposium, Reyjkjavik, 2006, p. $130-33$.
56 Eysenck MW, Derakshan N, Santos R, Calvo MG. Anxiety and cognitive performance: attentional control theory. Emotion. 2007 May;7(2):336-53

57 Greenwood LM, Broyd SJ, Croft R, Todd J, Michie PT, Johnstone S, et al. Chronic effects of cannabis use on the auditory mismatch negativity. Biol Psychiatry. 2014 Mar; 75(6):449-58.

58 Rentzsch J, Buntebart E, Stadelmeier A, Gallinat J, Jockers-Scherübl MC. Differential effects of chronic cannabis use on preattentional cognitive functioning in abstinent schizophrenic patients and healthy subjects. Schizophr Res. 2011 Aug;130(1-3):222-7.

59 Lee M, Hoptman M, Sehatpour P, Lakatos P, Dias E, Kantrowitz J, et al. Neural mechanisms of mismatch negativity (MMN) dysfunction in schizophrenia. Int J Psychophysiol. 2016;108:37.

60 Kathmann N, Frodl-Bauch T, Hegerl U. Stability of the mismatch negativity under different stimulus and attention conditions. Clin Neurophysiol. 1999 Feb;110(2):31723.

61 Argyle N, Deltito J, Allerup P, Maier W, Albus $\mathrm{M}$, Nutzinger $\mathrm{D}$, et al. The Panic-Associated Symptom Scale: measuring the severity of panic disorder. Acta Psychiatr Scand. 1991 Jan;83(1):20-6. 